\title{
Wideband delays generated in an all-optical tunable delay line, preserving signal wavelength and bandwidth
}

\author{
Luc Thévenaz*, Sanghoon Chin \\ École Polytechnique Fédérale de Lausanne, Institute of Electrical Engineering, STI-GR-SCI-LT Station 11, CH-1015 Lausanne, Switzerland
}

Available online 31 December 2009

\begin{abstract}
A novel technique to produce large all-optically controlled tunable delays of $100 \mathrm{ps}$ pulse train in optical fibers is demonstrated. The configuration of the delay line basically consists of two main stages: the wavelength conversion via semiconductor optical amplifiers and the group velocity delaying via a dispersive optical medium. The wavelength-converted signal was precisely delayed over a wide temporal range from picoseconds to nanoseconds using a dispersive fiber, preserving the wavelength and the bandwidth of the signal. 100 ps FWHM signal pulses were delayed continuously up to 14 ns with moderate pulse distortion, corresponding to a 140-bit delay. To cite this article: L. Thévenaz, S. Chin, C. R. Physique 10 (2009).
\end{abstract}

๑) 2009 Académie des sciences. Published by Elsevier Masson SAS. All rights reserved.

\section{Résumé}

Retards à grande bande passante engendrés dans une à ligne à retard tout-optique accordable, préservant la longueur d'onde et la bande passante du signal. Une nouvelle technique pour produire des grands retards ajustables et contrôlés de manière tout-optique, cesi pour un train d'impulsions de $100 \mathrm{ps}$ dans des fibres optiques, est ici démontrée. La ligne à retard est réalisée par la combinaison de deux fonctions principales : une conversion de longueur d'onde via des amplificateurs optiques à semiconducteurs et un retardement du signal via un milieu optique dispersif. Le signal converti en longueur d'onde a été retardé avec précision par propagation dans une fibre dispersive sur une échelle temporelle allant de la picoseconde à quelques nanosecondes, en conservant la longueur d'onde et la bande passante du signal. Des impulsions de 100 ps ont été retardé continûment jusqu'à $14 \mathrm{~ns}$ avec une distorsion réduite, correspondant à un retard séquentiel de 140-bits. Pour citer cet article $:$ L. Thévenaz, S. Chin, C. R. Physique 10 (2009).

(C) 2009 Académie des sciences. Published by Elsevier Masson SAS. All rights reserved.

Keywords: Optical fibers; Nonlinear optics; Wavelength conversion; Optical propagation in dispersive media; Semiconductor optical amplifier; Slow light

Mots-clés : Fibres optiques ; Optique non-linéaire ; Conversion en longueur d'onde ; Propagation optique dans milieu dispersif ; Amplificateur optiques à semi-conducteurs; Lumière lente

\footnotetext{
* Corresponding author.

E-mail address: luc.thevenaz@epfl.ch (L. Thévenaz).
} 


\section{Introduction}

The development of all-optically controlled delay lines has been one of the greatest challenges for photonic signal processing since they provide the required fast timing function in advanced optical signal processing and they are believed necessary for the development of the future all-optical packet routers in photonic communication systems. Slow and fast light has been proposed as one potential approach using various physical phenomena [1,2]. Besides, the possibility to generate slow light in optical fibers using stimulated scatterings [3-7] or parametric processes [8] led to a significant progress towards real applications since such slow light systems have the advantages of the compatibility with fiber-optic communication systems, a room-temperature operation at any wavelength and a large data bandwidth. However, it was soon identified that the maximum time delay that signal pulses can experience is practically restricted to a few pulse-width delays in all slow light systems, so that the delay-bandwidth product that can be achieved in active slow light systems is approximately equal to unity. It is mainly due to the large pulse distortion resulting essentially from the spectral filtering effect in the narrow band resonance and the nonlinear phase dependence on frequency in the slow light medium [9]. Therefore, the scientific challenge to break the unity delay-bandwidth product and thus to produce a large amount of pulse delay with negligible distortion remains opened.

To overcome this issue, an elegant solution was recently proposed using the combination of wavelength conversion and group velocity dispersion [10-12], resulting in significant pulse delays for broadband signals. In this article, we propose a modified configuration of this technique with the goal of making an optical delaying system as transparent as possible for the signal, i.e. preserving the temporal sequence, the signal wavelength and its bandwidth. A key advantage in our configuration compared to previous works is to realize simply and efficiently the wavelength conversion with a compact and low power concept using cross-gain modulation in a semiconductor optical amplifier. It must be pointed out that this type of conversion turns out to be efficient over a much broader wavelength range than parametric processes. This extends the range of delays obtained by the dispersive line and we could demonstrate experimentally a large range of optical delays up to tens of nanosecond for 100 ps optical pulses with low distortion.

\section{Principle}

Cross-gain modulation (XGM) in a semiconductor optical amplifier (SOA) is usually described as a nonlinear interaction between two co- or counter-propagating beams, a strong pump wave at frequency $v_{\text {pump }}$ and a weak probe wave at frequency $v_{\text {probe }}$. Let suppose an intensity modulated pump light entering an SOA and a continuous wave (CW) probe light simultaneously injected counter-directionally into the SOA, as depicted in Fig. 1. Due to the gain saturation, the pump light will modulate the gain inside the amplifier [13]. In turn XGM in the amplifier will impose the pump modulation onto the probe. Consequently, the intensity of the probe at the target wavelength is inversely modulated and then carries a complementary data pattern, so essentially the same information as the pump modulation. This way the wavelength of the signal can be converted with high efficiency and no requirement for strict phase matching conditions, which remains necessary to obtain parametric conversions [10-12]. Moreover, this process can be polarization-independent if an SOA showing a polarization-independent gain is employed.

After the first wavelength conversion is accomplished, the probe light exiting from the SOA at the converted wavelength is delivered into a highly dispersive optical medium such as long lengths of optical fiber [10,11] or even a highly dispersive Bragg grating [12]. The propagation velocity of the probe through the dispersive fiber is continuously varied by simply tuning the probe wavelength as a result of the wavelength dependence of the group velocity. This way, the probe wave can exit the dispersive fiber with relative temporal delays or advancements. The amount of time delay achievable through this delay line can be simply estimated to first order as the product of probe wavelength change and the average group-velocity dispersion (GVD) in the fiber. The delayed converted signal then experiences a second wavelength conversion through another SOA back to the original signal wavelength, with the further advantage to restore the original modulation pattern through the same process.

\section{Experiments and results}

The schematic diagram of the experimental setup is depicted in Fig. 2. A commercial distributed feedback (DFB) laser diode operating at $1571 \mathrm{~nm}$ was used as a light source and its output was split by a 90/10 directional coupler. The higher power branch was optically gated using a fast electro-optic Mach-Zehnder intensity modulator (EOM) to 


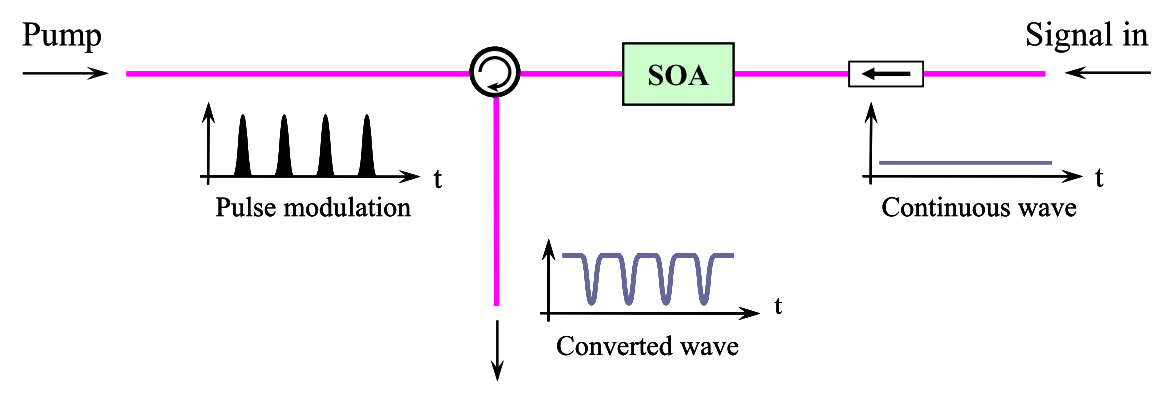

Signal out

Fig. 1. Schematic diagram of the principle to generate a wavelength conversion through cross-gain modulation (XGM), in the particular situation in which the pump and signal waves counter-propagate through the semiconductor optical amplifier.

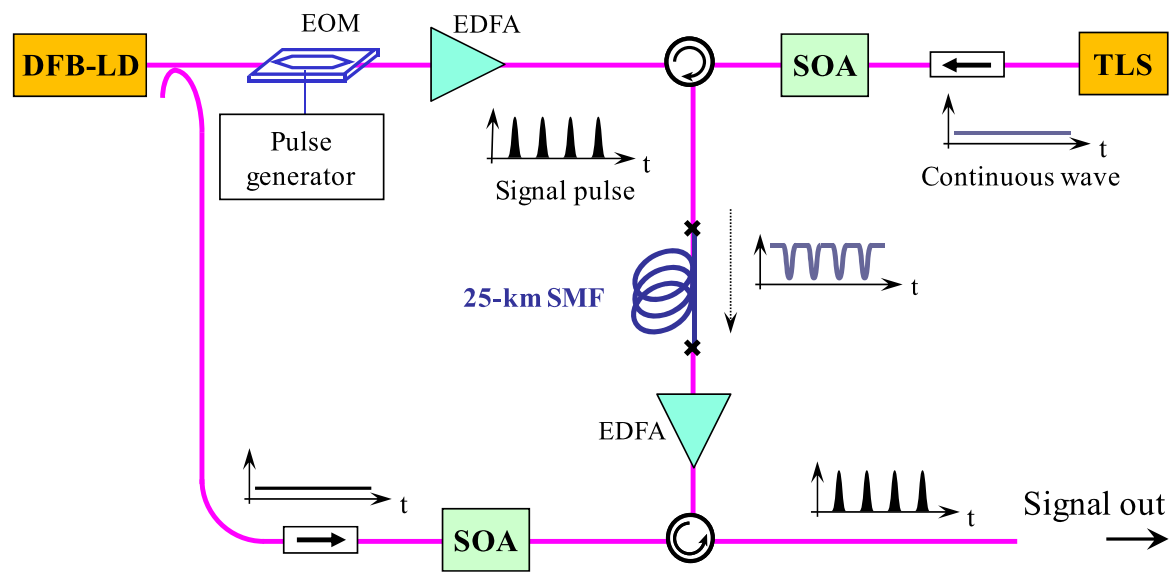

Fig. 2. Schematic configuration of the experimental setup to realize a transparent tunable all-optical delay line. EOM, electro-optic modulator; SOA, semiconductor optical amplifier; TLS, tunable laser source.

produce a signal pulse train with duration of $100 \mathrm{ps} \mathrm{FWHM}$ at a repetition rate of $500 \mathrm{MHz}$. Then the pulse train was boosted using an erbium-doped fiber amplifier (EDFA) before entering into the first SOA, so that it could play the role of the pump in the first wavelength conversion process. A weak continuous wave $(\mathrm{CW})$ probe beam at a target wavelength was generated by a tunable laser source (TLS) and was also simultaneously launched into the SOA, but in the direction opposite to the input pulse direction. This way the converted probe signal could be simply separated from the input pump pulse without any need of optical filters. According to the principle described above, XGM in the amplifier induces an intensity modulation onto the probe, resulting in a wavelength converted signal showing inverted intensity levels compared to the modulation pattern of the input pulses. The converted signal was then delivered to a dispersive medium to experience a relative time delays. In our setup, it was simply a $25 \mathrm{~km}$-long non-zero dispersionshifted single-mode fiber (NZ-DSF), showing an accumulated group velocity dispersion of $\sim 180 \mathrm{ps} / \mathrm{nm}$. After exiting from the dispersive fiber, the wavelength converted signal was amplified using another EDFA before entering into the second SOA. The lower power channel of the initial DFB laser was also launched into the SOA to play the role of the probe in the second wavelength conversion. Consequently, the original pattern of the signal pulse is retrieved through a second pattern inversion and the signal returns to its original wavelength.

The normalized time waveforms of the signal pulses exiting from the delay line were measured and displayed on a digital oscilloscope for different wavelength of the TLS, as shown in Fig. 3. The pulse delays are clearly observed without significant broadening while the TLS wavelength is increased by $2 \mathrm{~nm}$ steps. It must be mentioned that the on-off ratio of the sequence is degraded by the two consecutive conversions, since the SOAs are never totally saturated and thus do not turn fully blocking. This effect is not apparent in Fig. 3, the detector containing a DC block capacitor that suppresses the offset due to the background light present during the "off" sections. The alternate saturation in the two consecutive wavelength conversions limits the impact of the amplified spontaneous emission (ASE) to the 


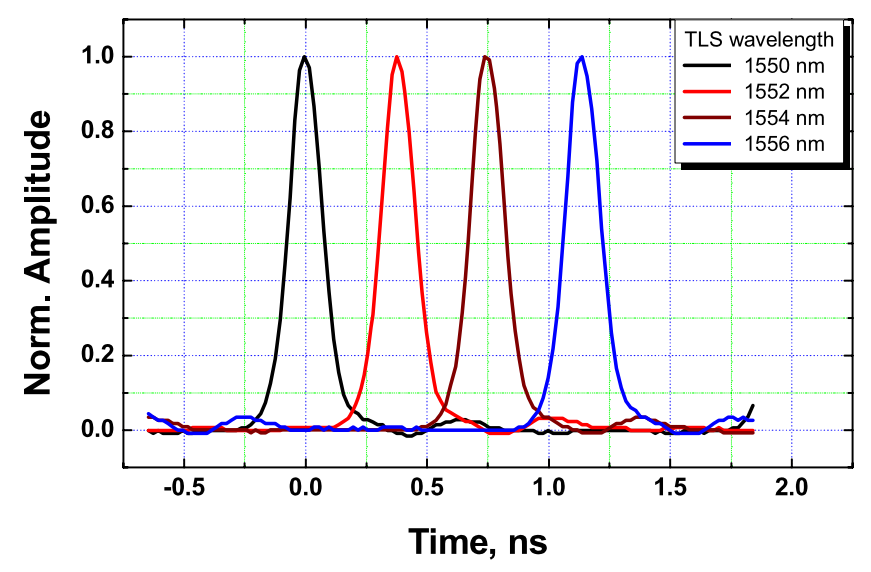

Fig. 3. Time waveforms of the delayed signal pulses while the wavelength of tunable laser source was swept from $1550 \mathrm{~nm}$ to $1556 \mathrm{by} 2 \mathrm{~nm}$ steps, showing the low distortion induced by the delaying scheme.

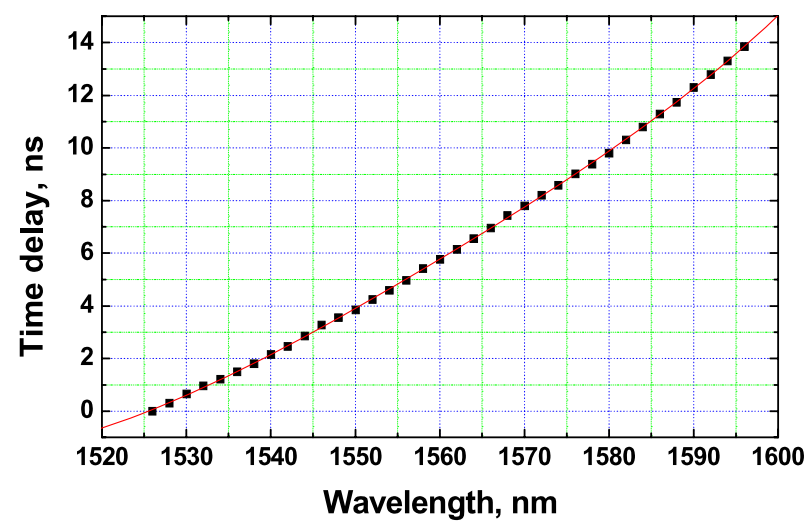

Fig. 4. Relative pulse delays as a function of the wavelength of the tunable laser source. The solid curve represents a 4th order polynomial fit.

equivalent of a single stage: ASE is critical only when the SOA is not saturated, hence when it is not blocking. In the second SOA the saturating signal comes from the first conversion in the first SOA and its ASE content is thus not transmitted to the final wavelength-converted output signal. For this reason the double conversion does not show a significantly worse optical signal-to-noise ratio than a single conversion using XGM and the added noise remains at a reasonable level.

To determine the amount of signal delay we used the peak value of the pulse. The largest achieved time delay was $14 \mathrm{~ns}$ corresponding to a fractional delay of 140 while the TLS wavelength was swept from $1526 \mathrm{~nm}$ to $1596 \mathrm{~nm}$, as illustrated in Fig. 4. This ratio can certainly be further improved using a more dispersive delay line and an even broader wavelength scanning range.

It must be pointed out that the delay lines based on wavelength conversion and group velocity dispersion also suffer from the trade-off between maximum delay and associated pulse distortion expressed by broadening. Since the chromatic dispersion is the quantity controlling the amount of time delay, the signal must experience the classical broadening due to group velocity dispersion when it travels through the medium, limiting the signal bandwidth. It should be mentioned that solutions can alleviate this limitation, for instance by imposing an extrinsic frequency chirp across a pulse signal or, as recently experimentally demonstrated by Okawachi et al., by completely compensating the large induced pulse broadening using phase conjugation through 4-wave mixing [14].

In order to achieve 1000-bit delay, we calculated the anticipated broadening of a transform limited Gaussian shaped pulse with duration of $100 \mathrm{ps}$ FWHM as a function of the GVD integrated over the length of the dispersive element and the required wavelength change [15], as shown in Fig. 5. According to this simple model, our delay line, showing 


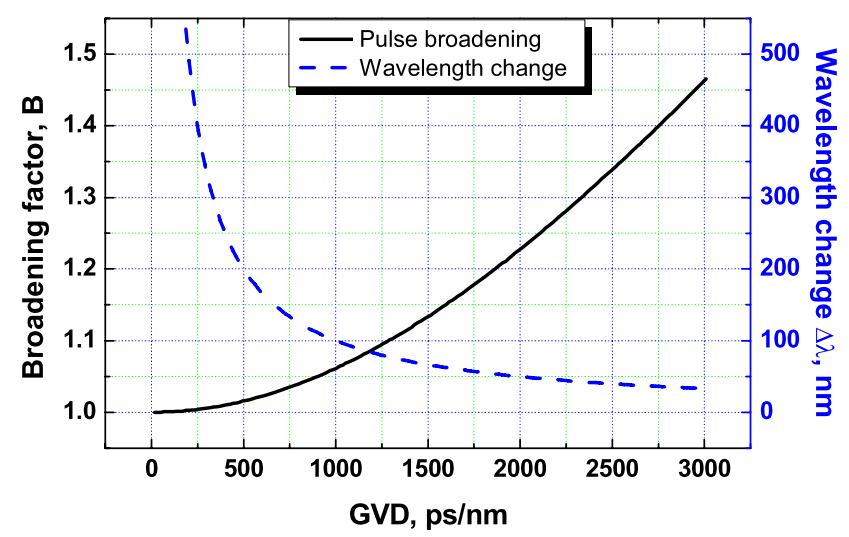

Fig. 5. Associated pulse broadening and the required wavelength change in order to produce 1000-bit delay for a 100 ps bit duration, estimated as a function of the accumulated group velocity dispersion (GVD) in the dispersive line, using a simple linear model applied to a coherent transformlimited Gaussian pulse.

a total GVD of $180 \mathrm{ps} / \mathrm{nm}$, requires a wavelength change of $\sim 550 \mathrm{~nm}$ to produce a fractional delay of 1000 , which is unrealistic in real operational conditions. An alternative way is to increase the total GVD and Fig. 5 shows that a GVD larger than $1000 \mathrm{ps} / \mathrm{nm}$ is necessary to realize the 1000-bit delay with a wavelength change smaller than $100 \mathrm{~nm}$. The broadening remains acceptable if GVD does not exceed $2000 \mathrm{ps} / \mathrm{nm}$. It must be mentioned that this represents ideal figures for an unchirped transform-limited pulse and the XGM process certainly superimposes a phase modulation (and thus a frequency chirp) on the intensity pattern.

\section{Conclusions}

We have experimentally demonstrated the generation of large variable optical delays for $10 \mathrm{Gbit} / \mathrm{s}$ data streams, based on the combination of GVD in optical fibers and double wavelength conversion using XGM in SOA. In comparable conditions in terms of bandwidth and optical powers this approach widely surpasses implementations based on slow light using a spectral resonance. When compared to similar techniques using other processes for wavelength conversion - such as those based on 4-wave mixing and self-phase modulation - our scheme presents the advantage to require moderate optical powers and to operate over a wide spectral range $(\sim 100 \mathrm{~nm})$. Moreover, the original signal wavelength can be fully preserved while producing optical delays; nevertheless the phase information is lost in the process, meaning that the technique remains restricted to pure amplitude-based modulation coding (ASK) and to digital signals only. It must be pointed out that the signal spectrum can be moderately altered by a possible chirp effect while switching to gain saturation in the SOAs [16]. Even though a real data stream was not used for this demonstration it looks quite evident that this scheme is very immune to the actual time ordering of the bit sequence if the probe intensity is kept sufficiently low in the XGM process. In this experiment, the maximum bit-rate of the data packet through the delay-line was restricted by the carrier recovery time of the available SOAs. However, it must be pointed out that our scheme can potentially be applied for high speed networks since error-free wavelength conversion at $160 \mathrm{Gbit} / \mathrm{s}$ has been realized using a similar process [17]. This makes us confident that this scheme can be a simple and performing timing tool for future communication and microwave photonics systems.

\section{Acknowledgement}

The authors would like to acknowledge the support from the Swiss National Science Foundation through project 200020_121860 and the European Community's Seventh Framework Programme [FP7/2007-2013] under grant agreement no. 219299 (GOSPEL project).

\section{References}

[1] R.W. Boyd, D.J. Gauthier, 'Slow' and 'Fast' light, in: E. Wolf (Ed.), Progress in Optics, vol. 43, Elsevier, Amsterdam, 2002, pp. 497-530, Ch. 6. 
[2] J.B. Khurgin, R.S. Tucker, Slow Light: Science and Applications, CRC Press, Boca Raton, 2009.

[3] K.Y. Song, M. Gonzalez-Herráez, L. Thévenaz, Observation of pulse delaying and advancement in optical fibers using stimulated Brillouin scattering, Opt. Express 13 (2005) 82-88.

[4] Y. Okawachi, M.S. Bigelow, J.E. Sharping, Z. Zhu, A. Schweinsberg, D.J. Gauthier, R.W. Boyd, A.L. Gaeta, Tunable all-optical delays via Brillouin slow light in an optical fiber, Phys. Rev. Lett. 94 (2005) 153902.

[5] M. Gonzalez-Herráez, K.Y. Song, L. Thévenaz, Optically controlled slow and fast light in optical fibers using stimulated Brillouin scattering, Appl. Phys. Lett. 87 (2005) 081113-1-081113-3.

[6] L. Thevenaz, Slow and fast light in optical fibres, Nat. Photon. 2 (8) (2008) 474-481.

[7] J.E. Sharping, Y. Okawachi, A.L. Gaeta, Wide bandwidth slow light using a Raman fiber amplifier, Opt. Express 13 (2005) $6092-6098$.

[8] D. Dahan, G. Eisenstein, Tunable all optical delay via slow and fast light propagation in a Raman assisted fiber optical parametric amplifier: a route to all optical buffering, Opt. Express 13 (2005) 6234-6249.

[9] M. Gonzalez-Herráez, L. Thévenaz, Physical limits to broadening compensation in linear show light systems, Opt. Express 17 (2009) $4732-$ 4739.

[10] J.E. Sharping, Y. Okawachi, J.V. Howe, C. Xu, Y. Wang, A.E. Willner, A.L. Gaeta, All-optical, wavelength and bandwidth preserving, pulse delay based on parametric wavelength conversion and dispersion, Opt. Express 20 (2005) 7872-7877.

[11] Y. Okawachi, J.E. Sharping, C. Xu, A.L. Gaeta, Large tunable optical delay via self-phase modulation and dispersion, Opt. Express 14 (2006) 12022-12027.

[12] M. Fok, C. Shu, Tunable pulse delay using four-wave mixing in a 35-cm bismuth oxide highly nonlinear fiber and dispersion in a chirped fiber Bragg grating, in: Proc. ECOC'06, Cannes, France, 2006.

[13] T. Durhuus, B. Mikkelsen, C. Joergensen, S.L. Danielsen, K.E. Stubkjaer, All-optical wavelength conversion by semiconductor optical amplifiers, J. Lightwave Technol. 14 (1996) 942-954.

[14] Y. Okawachi, M.A. Foster, X. Chen, A.C. Turner-Foster, R. Salem, M. Lipson, C. Xu, A.L. Gaeta, Large tunable delays using parametric mixing and phase conjugation in Si nanowaveguides, Opt. Express 16 (2008) 10349-10357.

[15] G.P. Agrawal, Fiber-Optic Communication System, 3rd ed., John Wiley \& Sons, Inc., New York, 2002.

[16] G.P. Agrawal, N.A. Olsson, Self-phase modulation and spectral broadening of optical pulses in semiconductor laser amplifiers, J. Quantum Electron. 25 (1989) 2297-2306.

[17] Y. Liu, E. Tangdiongga, Z. Li, S. Zhang, H. Waardt, G.D. Khoe, H.J. Dorren, Error-free all-optical wavelength conversion at 160 Gb/s using a semiconductor optical amplifier and an optical bandpass filter, J. Lightwave Technol. 24 (2006) 230-236. 